\title{
A 60-year-old man with bilateral painful reticular patches of violaceous colour on the soles
}

\author{
Burak TEKİN, Merve Hatun SARIÇAM, Züleyha ÖZGEN, Cuyan DEMİRKESEN, Zeynep KOMESLİ, İzzet Hakkı ARIKAN
}

A 60-year-old male patient was referred for the evaluation of skin lesions on his soles which started as violaceous macules on tips of his toes three weeks before admission. Later, the lesions slowly progressed proximally to involve most of his soles in a mottled pattern and became painful. Physical examination revealed bilateral tender dark violaceous patches of reticular configuration on the soles, more prominent on the plantar surfaces of the distal phalanges of the right foot (Figure 1). The reticular patches partly extended to the dorsal parts of the feet. A darker macule with a $0,5 \times 1 \mathrm{~cm}$ sized eroded and necrotic area was seen on the plantar surface of the fifth digit of the right foot (Figure 2). Both feet were exquisitely tender to palpation. Dorsal pedal pulses were palpable bilaterally. Routine laboratory analysis revealed elevated blood urea nitrogen $(61 \mathrm{mg} / \mathrm{dl}$; normal range: $5-25 \mathrm{mg} / \mathrm{dl})$, serum creatinine $(4,33 \mathrm{mg} / \mathrm{dl}$; normal range: $0,5-1,10 \mathrm{mg} / \mathrm{dl})$ and $\mathrm{C}$-reactive protein levels $(7,5$ $\mathrm{mg} / \mathrm{dl}$ normal range: $0-5 \mathrm{mg} / \mathrm{dl}$ ). The patient had a 6-year history of hypertension and a 4-year history of diabetes mellitus which had been managed with antihypertensives and oral antidiabetics, respectively. His medical history was also remarkable for a coronary artery bypass graft ( 25 years ago), a transient left upper extremity weakness ( 2 years ago) and right carotid artery stent ( 7 months ago). Three months ago, he was admitted by the Neurology Department for acute-onset paraplegia and slurred speech and was diagnosed as a cerebrovascular accident. During his hospitalization in neurology, a nephrology consultation was obtained due to persistently elevated serum creatinine levels $(2,10$ to $2,69 \mathrm{mg} / \mathrm{dl}$; normal range: $0,5-1,10 \mathrm{mg} / \mathrm{dl}$ ) and the patient

Burak Tekin ( $\varangle)$, Merve Hatun Sariçam, Züleyha Özgen

Department of Dermatology, School of Medicine, Marmara University, Istanbul, Turkey

e.mail:buraktekin@hotmail.com

Cuyan Demirkesen

Department of Pathology, Cerrahpaşa School of Medicine, Istanbul University, Istanbul, Turkey

Zeynep Komesli

Department of Internal Medicine, School of Medicine, Marmara University, Istanbul, Turkey

İzzet Hakkı Arıkan

Sub-department of Nephrology, Department of Internal Medicine, School of

Medicine, Marmara University, Istanbul, Turkey
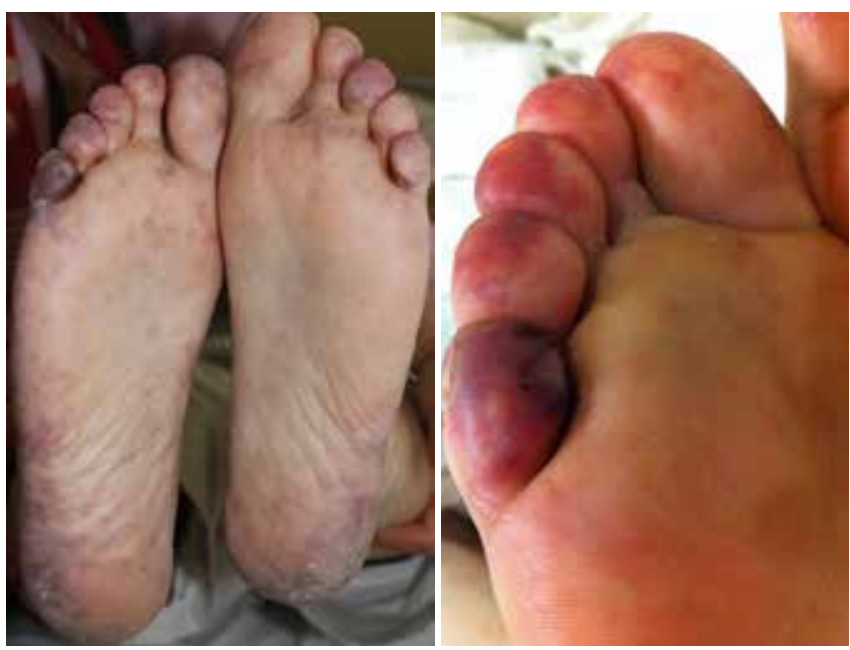

Figure 1. Reticular violaceous macules Figure 2. A necrotic area on the fifth on the plantar surfaces of both feet.

digit of the right foot.

was diagnosed with chronic renal failure. Anticoagulant treatment with warfarin and enoxaparin was initiated by the cardiologists for atrial fibrillation. The patient denied smoking, alcoholism or any illicit drug abuse. A $4 \mathrm{~mm}$ deep punch biopsy was taken from the reticular patches on the lateral side of the right foot (Figure 3).

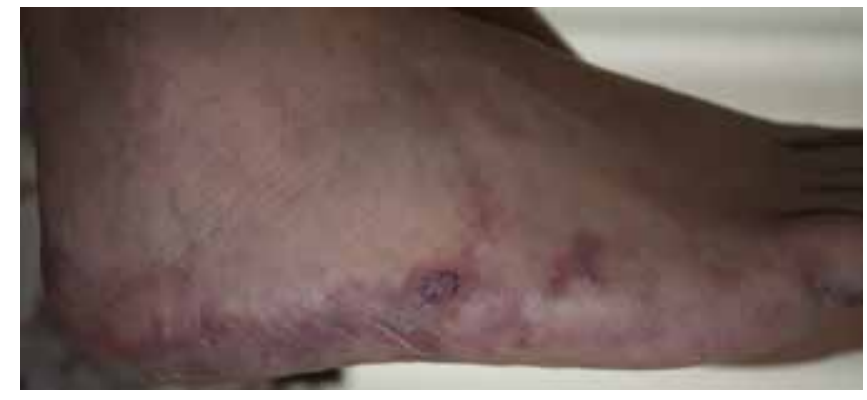

Figure 3. Reticular violaceous macules extending to the dorsum of the right foot. Punch biopsy was taken from the circled area.

\section{What is your diagnosis?}
A) Calciphylaxis
B) Microscopic polyangiitis
C) Cholesterol embolism
D) Cryoglobulinemia
E) Polyarteritis nodosa 


\section{ANSWER to PHOTO QUIZ}

\section{Cholesterol embolism}

\section{Discussion}

Cholesterol embolism (atheroembolism) is caused by embolization of cholesterol crystals from the major arteries to arteries of smaller caliber. Whereas this frequently unrecognized disease may be spontaneous, it usually occurs as an iatrogenic complication of invasive vascular procedures, thrombolytic treatment or anticoagulation that can all cause the removal of cholesterol crystals from already formed atheromatous plaques [1]. It is primarily a disease of men 50 years or older who have a history of atherosclerotic disease [2].

Because atheromatous plaques are most frequently located in the descending aorta, the kidneys and the skin of the lower extremities are most commonly affected. Typically, pulses are still palpable as small arterioles rather than arteries are affected [1]. Our patient had many well-known risk factors for cholesterol embolism, such as the male gender, age $>50$ years, hypertension, diabetes mellitus, invasive cardiovascular procedures and anticoagulant therapy. Our patient's right carotid artery stenting can be considered an important triggering factor for the embolization of cholesterol crystals to various organs since it was followed by a progressive deterioration of renal function, by a cerebrovascular accident and eventually by skin lesions within a time frame of seven months. Anticoagulant therapy that was started three months before the diagnosis of cholesterol embolism may have played a contributory role for the appearance of skin lesions. The frequency of cutaneous findings has been reported to range from $35 \%$ to $96 \%$. The most common cutaneous findings are livedo reticularis (49\%), gangrene (35\%) and cyanosis (28\%). Ulceration, nodules and purpura have also been reported [3]. The diagnosis is confirmed by a deep biopsy specimen demonstrating cholesterol clefts within arterioles. These cholesterol clefts represent needle-shaped spaces left after the cholesterol crystals are dissolved as the tissue is processed for histologic examination. Figure 4 shows the histopathology of the punch biopsy specimen taken from our patient. Skin, as one of the most commonly affected organs, plays a major role in the diagnosis of cholesterol embolism because of the importance of histopathological confirmation in the diagnosis [3]. Skin is an easily accessible organ for biopsy and the reticular purpuric patches are the first choice of biopsy when present [2].

Our patient's renal failure might have been caused by cholesterol emboli lodging in the renal microvasculature. Renal failure has been reported to be one of the most

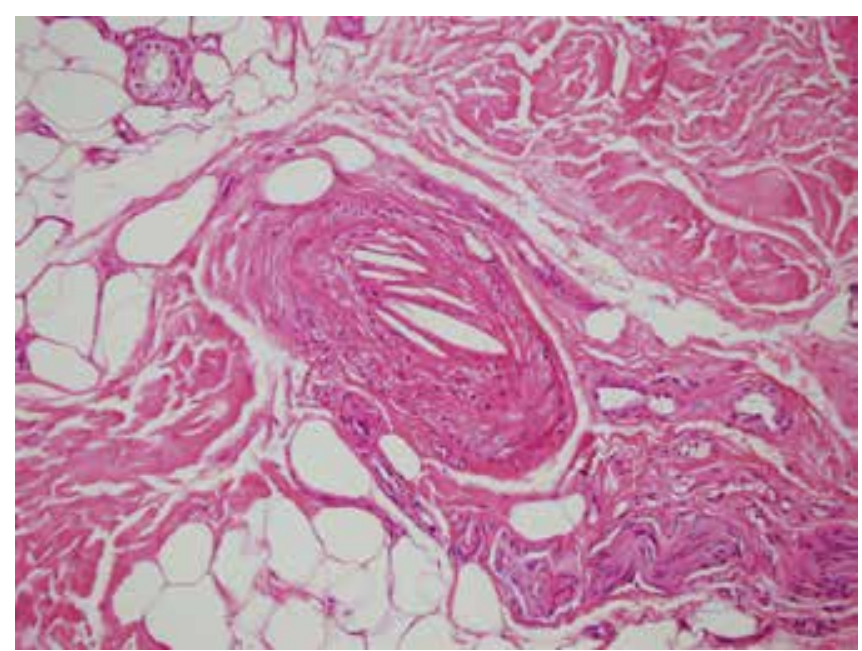

Figure 4. Obliteration of vascular lumen by cholesterol crystals (HEx200).

common findings on presentation, with up to one third of patients being affected [3]. The progressive increase in serum creatinine levels of our patient was not so high as to require haemodialysis. Apart from renal failure, a multisystemic presentation with a wide spectrum of clinical findings is also possible, including transient ischemic attacks, strokes, gastrointestinal ulcerations and bleeding, hemorrhagic pancreatitis and pulmonary involvement [2].

Treatment is mainly supportive. Anticoagulation should usually be discontinued. However, this decision should be given carefully weighing the risks and benefits of anticoagulation in every individual patient. Vascular procedures should generally be avoided. The recommended approach consists of treatment and prevention of cardiac failure and aggressive treatment of metabolic disorders using angiotensin converting enzyme inhibitors, loop diuretics, statins and haemodialysis [3,4]. Pentoxifylline has been reported anecdotally to be beneficial. The use of systemic corticosteroids to decrease antiinflamatory response is controversial. Surgery is generally not recommended and only reserved for select cases [3].

In our case, anti-inflammatory treatment with acetylsalicylic acid and methylprednisolone was commenced together with aggressive antihypertensive and analgesic treatment. Anticoagulant therapy was discontinued. The skin lesions partially resolved and the patient was discharged on the $13^{\text {th }}$ day after admission. The mortality rate of cholesterol embolism has been reported to be as high as $81 \%$ [3]. Though the initial management of our patient led to improvement, the long-term prognosis may be poor due to the presence of multiple ongoing risk factors.

In conclusion, a recent onset of livedo reticularis on the lower legs in elderly patients should raise the suspicion of cholesterol embolism. Skin biopsy of the livedoid reticular patches are helpful in confirming the diagnosis. 


\section{References}

1. Jucgla A, Moreso F, Muniesa C, Moreno A, Vidaller A. Cholesterol embolism: still an unrecognized entity with a high mortality rate. J Am Acad Dermatol 2006;55:786-93.

2. Piette WW. Cutaneous manifestations of microvascular occlusion syndromes. In: Bolognia JL, Jorizzo JL, Schaffer JV, editors.
Dermatology. 3rd ed. China: Elsevier, 2012:369-84.

3. Donohue KG, Saap L, Falanga V. Cholesterol crystal embolization: an atherosclerotic disease with frequent and varied cutaneous manifestations. J Eur Acad Dermatol Enereol 2003;17:504-11.

4. Belenfant X, Meyrier M, Jacquot C. Supportive treatment improves survival in multivisceral cholesterol crystal embolism. Am J Kidney Dis 1999;33:840-50. 